\title{
Cytological Studies in Some Dioecious Plants
}

\author{
By \\ Goichi Nakajima \\ Tochigi-ken Normal School, Utsunomiya
}

\section{Materials and Methods}

Materials used in this investigations are as follows:

Populus Sieboldii, Miq.

Salix Bakko, Kimura

S. Caprea, L.

S. grandulosa, Seem.

S. babylonica, L.

S. integra, Thumb.

S. sachalinensis, Fr. Schm.

Buchleya Joan, Makino

Humulus lupulus, L.

var. cordifolius, Maxim.

Cocculus trilobus, DC.
Clematis heracleaefolia, DC. Xanthoxylum piperitum, DC. Trichosanthes japonica, Regel.

T. cucumeroides, Maxim.

Carex grallatoria, Maxim.

Dioscorea gracillima, Miq.

D. Tokoro, Makino

Smilax hederacea, L. var. nipponica, Maxim.

S. Oldhami, Miq.

S. China, L.

Young anthers were taken from plants which were growing in wild and fixed with Carnoy's fluid excepting Trichosanthes cucumeroides for which Bouin's solution was used. The root tips were obtained from plants growing in pots with soil and fixed with Navashin's solution. All fixed materials were imbedded in paraffin, cut $12-20 \mu$ thick, and stained with Heidenhain's iron-alum-haematoxylin.

All figures are drawn by the aid of Abbe's large camera using Zeiss apochromatic objective $1.5 \mathrm{~mm}$ and Zeiss $\times 15$ compensation ocular. Magnification is $\times 2270$.

\section{Results}

Populus Sieboldii, Miq.: According to Blackburn and Harrison (1922, 1924), and Meurman (1925) the reduced chromosome numbers in male plants of $P$ tremula, $P$. trichocarpa, $P$. balsamifera, $P$. Simoni, and $P$. tremuloides are 19. In male plants of $P$. Sieboldii, Miq. I also observed 19 bivalent chromosomes at polar views in the metaphase of the first division (Fig. 1) and the same number of chromosomes were counted in the second metaphase. 
The chromosomes varies in size and one of them is particularly large. In side views in 1st metaphase I observed one unequal chromosome pair consisting of two chromosomes of different size and one of them is about twice as large as the other as shown in Fig. 2.

Salix: According to Blackburn and Harrison (1924), Harrison (1926) and Sinoto $(1925,1928,1929)$, the reduced chromosome numbers in Genus Salix are usually 19, 38 and 57 showing a polyploidy, while the numbers 22 and 44 were also observed by Blackburn and Harrison in some species and ca. 24 by Sinoto (1929) in $S$. sachalinensis forma Hokkaido. These investigators found one unequal pair of chromosomes in the male individuals in each of the above stated polyploid species as well as in $S$. sachalinensis $f$. Hokkaidô.

I have examined the following 6 species of Salix i.e., S. Bakko, S. Caprea, S. grandulosa, S. babylonica, S. integra, and S. sachalinensis. The bivalent chromosomes, one of which being especially large, showed variation in size in the male plants of each species except $S$. babylonica, in which I could not count the number of meiotic chromosomes. S. Bakko, S. Caprea, S. grandulosa, S. integra, and S. sachalinensis have 19 gemini respectively.

I could not detect unequal pairs of chromosomes in polar views of the hetero-typic metaphase in the male plants of these 6 species, but in side views of the same stage one unequal pair of chromosomes was clearly observed as shown in Figs. 4, 6, 7, 9, 11, and 13.

Buchleya Joan, Makino: This plant is well known as a half parasitic shrub in Japan proper. In male plants the reduced number of chromosome was counted as 15 at metaphase in hetero- and homotypic divisions (Fig. 14). One unequal chromosome pair was observed in side views at heterotypic metaphase (Fig. 15). The two members of this pair differing in size disjoined in the heterotypic anaphase and passed to different poles, as shown in Fig. $16 \mathrm{a}, \mathrm{b}$.

Humulus lupulus, L. var. cordifolius, Maxim.: The chromosome numbers of Humulus lupulus which was reported by Tournois (1914), Winge $(1914,1929)$, and Sinotô $(1929)$ were $n=10$ and $2 n=20$.

In this variety I counted 20 chromosomes in root tip cells of the female plants (Fig. 17) and in the tapetum cells of male plants. In the metaphase of hetero- and homotypic divisions in the male plants 10 chromosomes were observed. The same number was reported for $H$. lupulus by the above stated 3 authors.

I could always observed at polar views of the heterotypic metaphase of the male plants of this variety that 8 gemini were located separated from each other, while 2 were connected longitudinally with each other at their ends. This is shown in Fig. 18. In the 
homotypic metaphase also, 8 chromosomes out of 10 are separated and the remaining 2 were connected to each other at their ends.

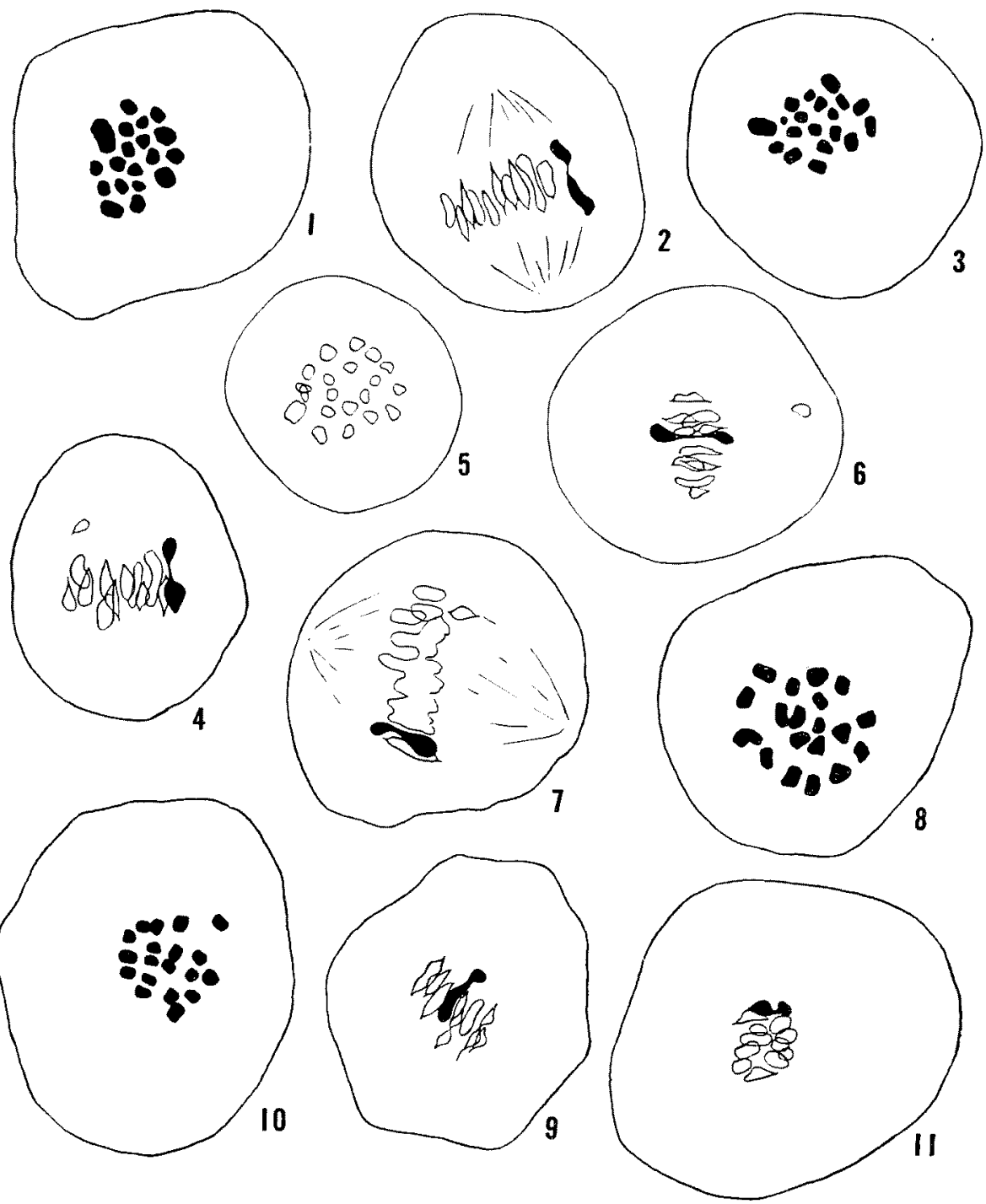

Figs. 1-2. Populus Sieboldii. 1, Polar views of heterotypic metaphase, showing 19 gemini. 2, Side view of the same stage; unequal pair of chromosomes in black. Figs. 3-4. Salix Bakko. 3, Polar view of heterotypic metaphase, showing 19 gemini. 4. Side view of the same stage; unequal pair of chromosomes in black. Figs. 5-6. S. glandulosa. 5, Polar view of heterotypic metaphase, showing 19 gemini. 6, Side view of the same stage; unequal pair of chromosomes in black. Fig. 7. S. babylonica. Side view of heterotypic metaphase; unequal pair in black. Figs. 8-9. S. Caprea. 8, Polar view of heterotypic retaphase, showing 19 gemini. 9. Side view of the same stage; unequal pair of chromosomes in black. Figs. 10-11. $S$. sachalinensis. 10, Polar view of heterotypic metaphase, showing 19 gemini. 11.

Side view of the same stage; unequal pair of chromosomes in black. 
I observed in the heterotypic metaphase of the male plants of this variety 8 gemini and one chromosome group consisting of 4 univalent chromosomes connected end to end. The same was reported in $H$. lupulus by Sinotô. In my material the 4 univalent chromosomes vary in their size and in side views of the heterotypic metaphase the group of 4 univalent chromosomes appears as a chain of $\mathrm{N}$ shape forming a tetrapartite chromosomes. A typical one is shown in Figs. 19, 21. The two middle members of the tetrapartite are larger than the end ones, and one of the end members is larger than the other. The large end ones are connected with a middle member more loosely than the other three. The same chromosome configuration was reported by Sinotô in $H$. lupulus. The 4 univalent chromosomes of the tetrapartite are named by Sinotô in $H$. lupulus as $\mathrm{X}_{1} \mathrm{Y}_{1} \mathrm{X}_{2} \mathrm{Y}_{2 .}$ in an order of their connection beginning with the large end chromosome to the smaller end one.

In my material in heterotypic anaphase $X_{1}$ and $X_{2}$ are separated from $Y_{1}$ and $Y_{2}$ and go to one pole together with the other 8 autosomes as the same in the cases of $H$. lupulus reported by Sinotô. The $\mathrm{X}_{1} \mathrm{X}_{2}$ and $\mathrm{Y}_{1} \mathrm{Y}_{2}$ chromosomes of the homotypic metaphase could be distinguished in their polar views from the autosomes (Fig. 20). In the heterotypic anaphase the tetrapartite chromosome occasionally goes toward a pole. The same behaviour of the tetrapartite was reported in H. lupulus by Sinotô. In such cases one of the daughter nuclei must contain all the chromosomes of the tetrapartite group. The cases in which the 4 elements are connected in the form of $\mathrm{X}_{1} \mathrm{Y}_{1} \vee / \mathrm{X}_{2} \mathrm{Y}_{2}$ or ${ }^{\mathrm{X}_{1}} \mathrm{Y}_{1} \mathrm{X}_{2} \vee \mathrm{Y}_{2}$ were also observed rarely. In such cases the allosomes may be separated in the heterotypic anaphase into two groups $X_{1} Y_{: 2}$ and $X_{2} Y_{1}$ or $X_{1} Y_{1}$ and $X_{2} Y_{22}$, and each of these groups may go to opposite poles together with the 8 autosomes (Fig. 22).

Cocculus trilobus, DC.: The reduced chromosome number of the male plants was found to be 25 at polar views in the heterotypic metaphase (Fig. 23). One unequal pair of chromosomes was observed in side views of the heterotypic metaphase. One of its two members is about twice as large as the other as shown in Fig. 24 in black. In some cells two or more laggards were observed.

Ciematis heracleaefolia, DC.: The writer (1933) found that the somatic number is 16 in the male plants of this species. In the present study 8 was determined as the reduced number at heterotypic metaphase in P.M.C's of the male plants (Fig. 25). But I could not observe unequal pair of chromosomes. A tetrad was observed which is consisted of 4 cells of various sizes. 

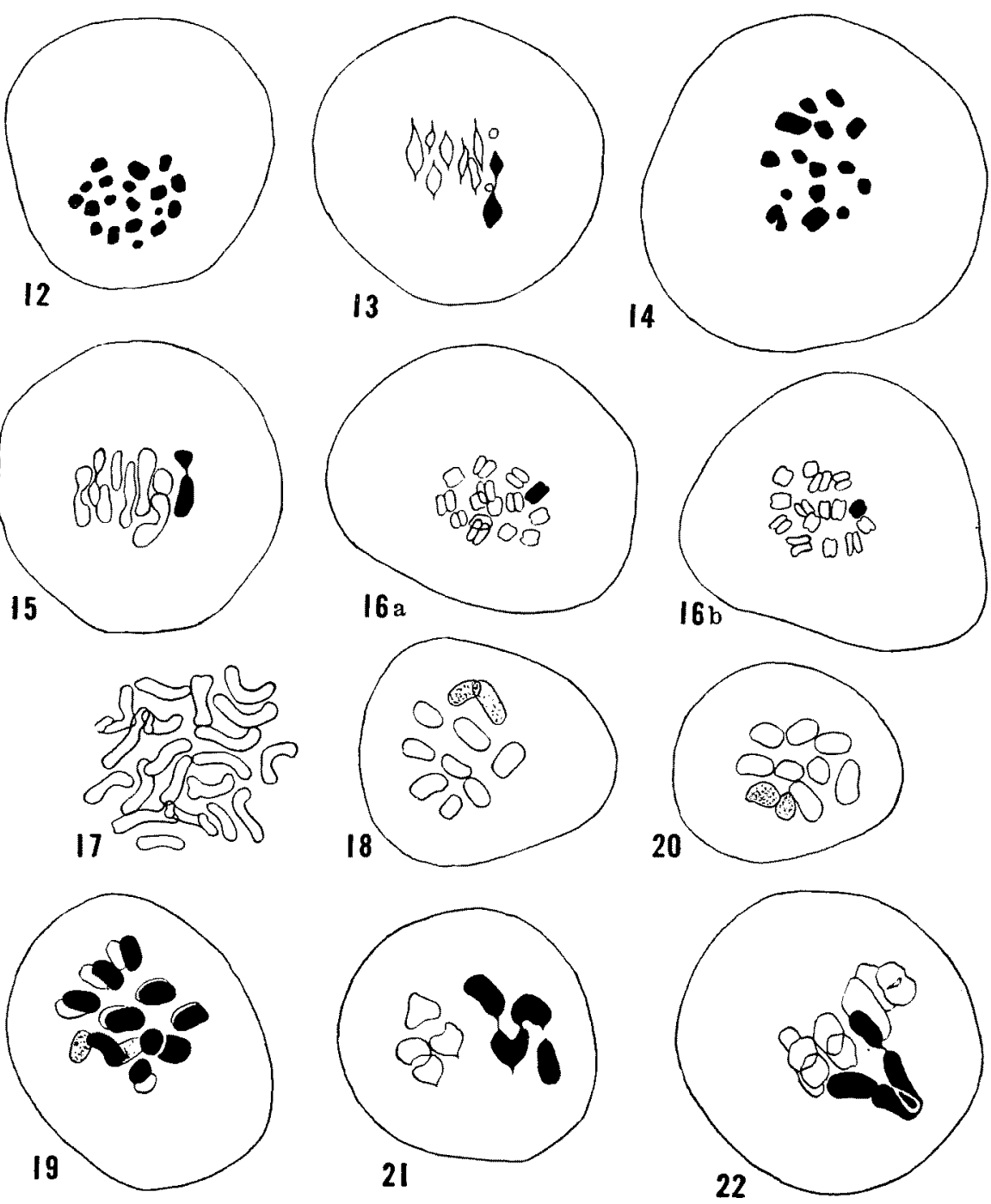

Figs.=12-13. S. integra. 12, Polar view of heterotypic metaphase, showing 19 gemini. 13, Side view of the same stage ; unequal pair of chromosomes in black. Figs. 1416. Buckleya Joan. 14, Polar view of beterotypic metaphase, showing 15 gemini. 15, Side view of the same stage ; unequal pair of chromosomes in black. $16 \mathrm{a}, \mathrm{b}$, Two chromosome groups in heterotypic anaphase; the two members of the unequal pair in black. Fig. 17. Somatic plate of female individuals of Humulus lupulus var. cordifolius. Figs. 18-22. Humulus lupulus var. cordifolius. 18, Polar view of heterotypic metaphase, showing 2 connection of $\mathrm{X}$ and $\mathrm{Y}$ gemini. 19, Polar view of meta-anaphase, showing one tetrapartite chromosome and 8 gemini. 20, Polar view of homotypic metaphase; dotted chromosomes are $\mathrm{X}$ and $\mathrm{Y}$. 21, Side view of heterotypic meta-anaphase; tetrapartite chromosome in black. 22, Abnormal behaviour of the tetrapartite chromosome in heterotypic division. 
Xanthoxylum piperitum, DC.: Sinoto (1929) reported in the male plants of this species that the reduced number is 35 and one of them was especially large and seemed to be univalent.

I counted also 35 chromosomes in polar views of the heterotypic metaphase of the male plant in the same species (Fig. 26). In side views in the same stage I observed one unequal pair of chromosomes (Fig. 27) and one univalent (Fig. 28). The univalent chromosome in Sinotô's material was large. On the contrary however it was small in my material. This occasionally reached the pole earlier than the others (Fig. 29). One or more laggards were observed and one or two chromosomes passed to the pole earlier than the others.

Trichosanthes japonica, Regel., $T$ cucumeroides, Maxim.: According to Sinotô (1928) and Sugimoto (1928) Trichosanthes presents, a polyploidy, the basic number being 11 . The chromosome number of $T$. japonica and $T$ cucumeroides were reported by Sinotô (1928) and Sugimoto (1928) to be $n=11,2 n=22$ and $n=22$, $2 n=44$ respectively.

The present writer also counted in the male plants 11 chromosomes of similar size in the heterotypic metaphase in $T$. japonica (Fig. 30) and 22 in the homotypic metaphase in $T$ cucumeroides (Fig. 32).

The observation in side views of the heterotypic metaphase showed the existence of one unequal pair of chromosomes in each species as shown in Figs. 31, 33.

Carex grailatoria, Maxim.: The reduced chromosome number was decided to be 9 in polar views of the heterotypic metaphase of the male plants (Fig. 34). The chromosomes vary in their size. One of them is especially larger than the others. I could not find any unequal pair in both polar and side views of the heterotypic division.

Dioscorea gracillima, Miq., D. Tokoro, Makino: According to Meurman (1925) the reduced numbers of chromosomes are 17 and 18 in Dioscorea sinuata and 10 in $D$. caucasca. In D. sinuata the type of sex chromosome was XO, while in D. caucasca the sex chromosomes were not observed. The somatic number of the male plants in these two species, $D$. gracillima and $D$. Tokoro, were reported by the present writer (1933) to be 20 in each. The reduced number in D. gracillima and D. Tokoro are 10 in each, corresponding to half that of their somatic ones (Figs. 35, 37). The gemini of D. gracillima varies in size, but those of $D$. Tokoro are of similar size. An unequal pair of chromosomes was observed in side views of the heterotypic metaphase in these two species (Figs. 36, 38). In the heterotypic anaphase of $D$. Tokoro the two members of the unequal pair were 

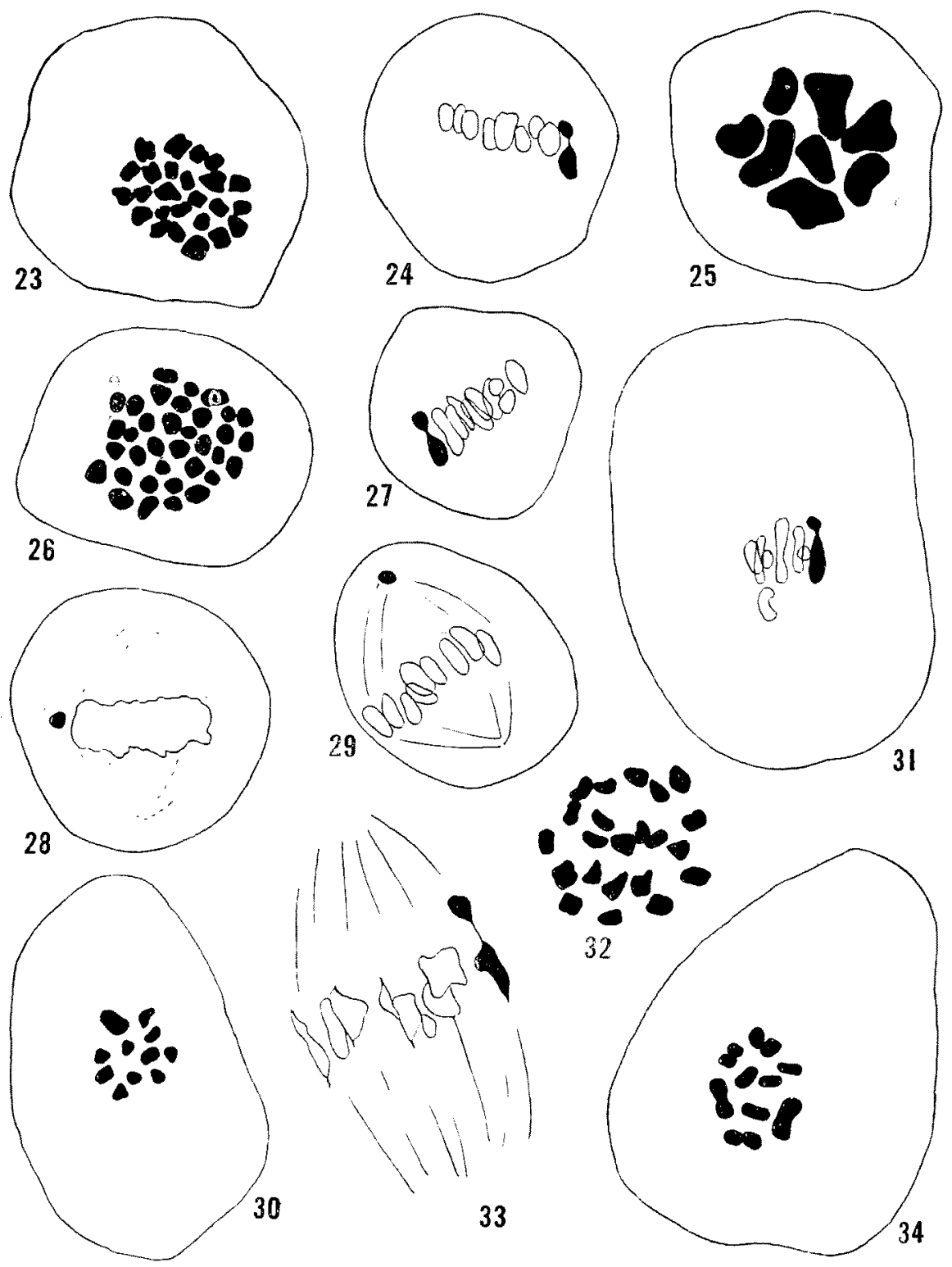

Figs. 23-24. Coccmlus trilobus. 23, Polar view of heterotypic metaphase, showing 25 gemini. 24, Side view of the same stage; unequal pair of chromosomes in black. Fig. 25. Clematis heraclecuerolia. Po'ar view of heterotypic metaphase, showing 8 gemini. Figs. 26-29. Xanthoxylum piperitum. 26, Polar view of heterotypic metaphase, showing 35 gemini. 27, Side view of the same stage; unequal pair of chromosomes in black. 28-29, Side views of the same stage, showing univalents in black. Figs. 30-31. Trichosanthes japonica. 30, Polar view of heterotypic metaphase, showing 11 gemini. 31, Side view of the same stage; unequal pair of chron osomes in black. Figs. 32-33. Trichosanthes cucumeroides. 32. Holar view of homotypic metaphase, showing 22 chromosomes. 33. Side view of heterotypic metaphase, unequal pair of chro nosomes in black. Fig. 34. Carex grallatoria. Polar view of heterotypic metaphase, showing 9 gemini. 
clearly observed to disjoin from each other and they passed to the opposite poles as shown in Fig. $39 \mathrm{a}, \mathrm{b}$.

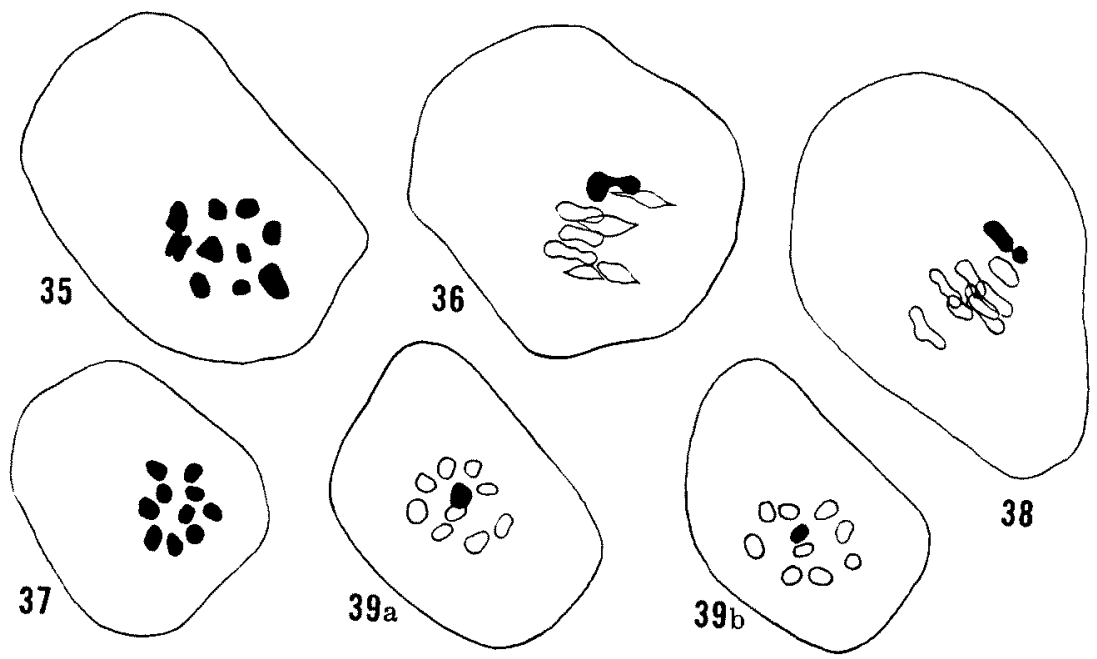

Figs. 35-36. Dioscorea gracillima. 35, Polar view of heterotypic metaphase, showing 10 gemini. 36, Side view of the same stage; unequal pair of chromosomes in black. Figs. 37-39. D. Tokoro. 37, Polar view of heterotypic metaphase, showing 10 gemini. 38, Side view of the same stage; unequal pair in black. $39 a, b, 2$ daughter nuclear plates in homotypic metaphase, showing the members of an unequal pair in black.

Smilax hederacea, L. var. nipponica, Maxim., S. Oldhami, Miq., S. China, L.: Cytological studies in Smilax herbacea were made by Humphrey (1914), Elkins (1914), and Lindsay (1929, 1930). According to the first two investigators the reduced number of the male plants was 12 , while Lindsay reported 13 . But the existence of sex-chromosomes was not recognized by them.

The present writer observed 30 rod shaped or curved somatic chromosomes of varying size in both male and female plants of $S$. hederacea, var. nipponica and one or two of them showed a satellite (Figs. 40, 41). The reduced number 15 of the male plants were counted in the polar view of the heterotypic metaphase (Fig. 42). In the side view an unequal pair of chromosomes was found (Fig. $43)$. In the heterotypic metaphase, one or two chromosomes passed to the poles earlier than the others.

In Smilax Oldhami, Miq. the somatic number 32 was counted in each of the 10 male and 2 female individuals. They were rod shaped or curved and their size varied (Figs. 44, 45). At diakinesis of P.M.C's one bivalent consisting of a large and a small members was observed (Fig. 46). In polar views of hetero- and homotypic metaphases 16 gemini of varying size were observed, one of which being 

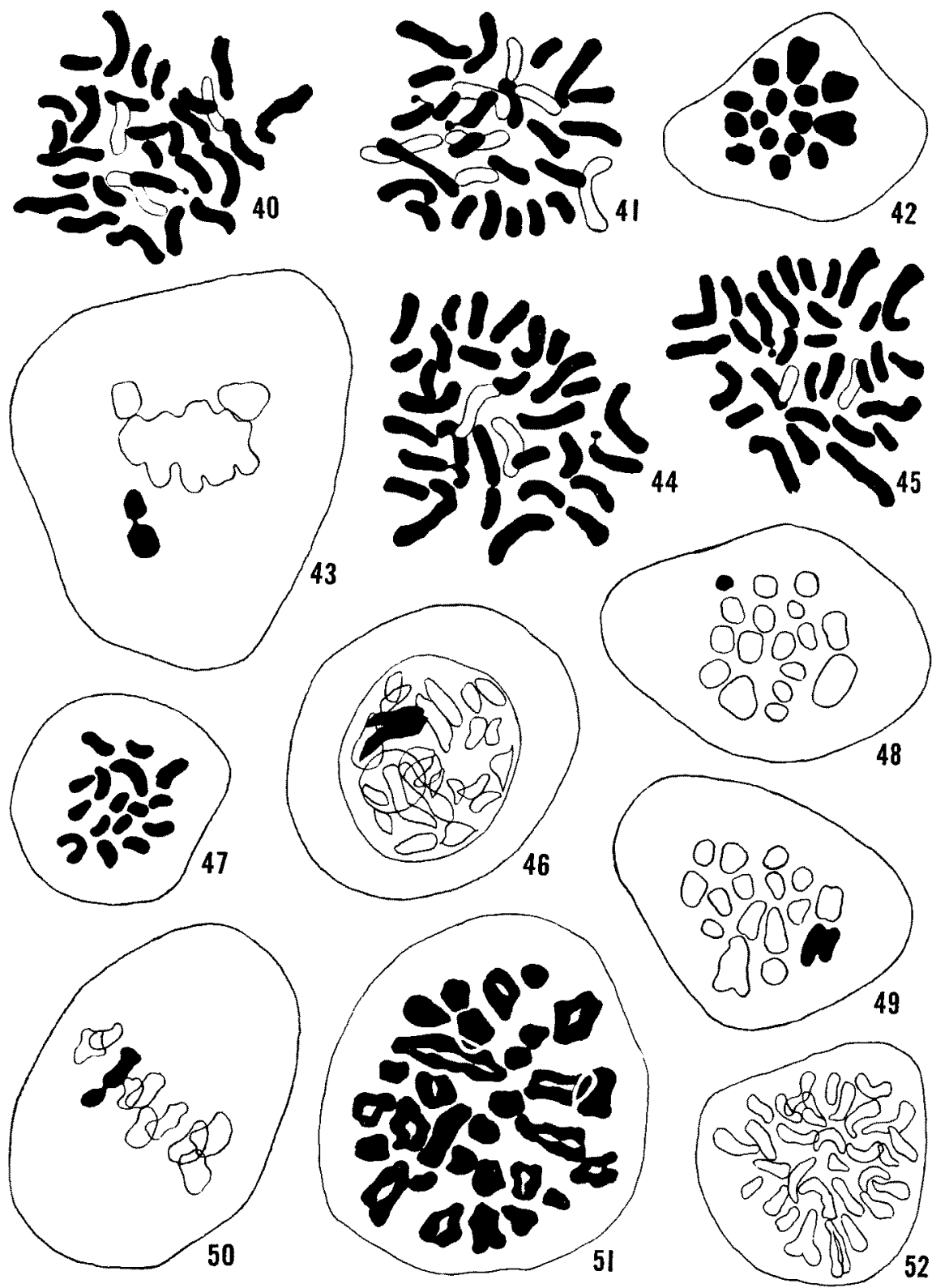

Figs. 40-43. Smilax hederacea, var. nipponica. 40, Male plant, showing 30 somatic chromosomes. 41, Female plant, showing 30 somatic chromosomes. 42, Polar view of heterotypic metaphase, showing 15 gemini. 43, Side view of the same stage; unequal pair of chromosomes in black. Figs. 44-50. Smilax Oldhami. 44, Male plants, showing 32 somatic chromosomes. 45, Female plants showing 32 somatic chromosomes. 46, Diakinesis, unequal pair in black. 47, Polar view of homotypic metaphase, showing 16 chromosomes. 48, Polar view of heterotypic metaphase, showing 16 gemini and a univalent chromosome in black. 49, Polar view of heterotypic metaphase, showing 16 gemini un€qual pair in black. 50, Side view of the same stage; unequal pair of chromosomes in black. Figs. 51-52. S. China. 51, Polar view of heterotypic metaphase, showing 30 gemini. 52, Polar view of homotypic metaphase, showing 30 chromosomes. 
especially large (Fig. 47). In the same stage a cell showing 16 chromosomes together with one small spheroid shaped chromosome which seems to be a univalent or a chromosome fragment was observed (black in Fig. 48). But the spheroid shaped chromosome could not be observed in somatic plates. In the heterotypic anaphase the univalent chromosome goes to the pole earlier or later than the others. In both polar and side views of the heterotypic metaphase I cbserved an unequal pair of chromosomes consisting of a large and a small members (black in Figs. 49,50). In the heterotypic anaphase up to 4 laggards were observed.

In Smilax China, L. 30 chromosomes of varying size were observed in polar views of the hetero- and homotypic metaphases in the male plants (Figs. 51,52). In side views of the heterotypic metaphase a heteromorphic pair was found the members of which differing in their size, were disjoining and passing to the poles earliar than the others.

In the heterotypic anaphase an unequal pair consisting of two large and small elements was observed and up to 3 laggards were observed there.

\section{Summary}

1) The male individuals of the dioecious plants comprising 11 genera, 18 species and 2 varieties have been investigated cytologically, with special reference to the sex chromosomes.

2) In each of 16 species and one variety one unequal pair of chromosomes was observed. This unequal pair is assumed to be a sex chromosome complex of XY type.

3) A tetrapartite chromosome was observed in Humulus lupulus var. cordifolius, which may be a sex chromosome complex of XYXY type.

4) In Carex grallatoria and Clematis heracleaefolia any morphological sex chromosome complex was not observed.

In closing the writer wishes to express his thanks to Prof. Kagawa of the Utsunomiya Agricultural College for his valuable suggestion in this work.

\section{Literature Cited}

Blackburn, K. B. (1926). On the occurrence of sex chromosomes in flowering plants with some suggestions as to their origin. Proc. of the Internat. Congr. of Plant Sci. 1.

- and Harrison, J. W. H. (1922). The meiotic phase in the Salicaceae. Rept. Brit. Ass. for the Adv. Sci., p. 398. cited from Blackburn (1929).

- and - (1924). A preliminary account of the chromosomes and chromosome behaviour in the Salicaceae. Ann. Botany 38.

Elkins, M. G. (1914). The maturation phase in Smilax herbacea. Bot. Gaz. 57. cited from Lindsay (1930). 
Harrison, J. W. H. (1926a). Heterochromosomes and polyploidy. Nature 117. cited from Blackburn (1929).

- (1929b). Polyploidy and sex chromosomes. Ib. 117. cited from Blackburn (1929).

Humphrey, L. E. (1914). A cytological study of the stamens of Smilax herbacea. Ohio Nat. 15. cited from Sinotô (1929).

Lindsay, R. H. (1930). The chromosomes of some dioecious angiosperms. Amer. Journ. Bot. 17.

Meurman, O. (1925). The chromosome behaviour of some dioecious plants and their relatives with special reference to the sex chromosomes. Soc. Sci. Fenn. Comm., Biol. 2.

Nakajima, G. (1933). Chromosome numbers in some angiosperms. Jap. Journ. Genet. 9.

Sinotô, Y. (1929). Chromosome studies in some dioecious plants, with special refarence to the allosomes. Cytologia 1.

Sugimoto, T. (1928). On the chromosome number in Cucurbitaceae. Rigakukai 26.

Tournois, J. (1914). Etudes sur la sexualité an haublon. Ann. Sci. Nat. 19. cited from Sinotô (1929).

Wettstein, F. v. (1927). Die Erscheinung der Heteroploidje, besonders im Pflanzenreich. Erg. d. Biolog. 2. cited from Sinotô (1929).

Winge, Ö. (1914). The pollination and fertilization process in Humulus lupulus L. and $H$. japonicus Sieb. et Zucc. C. R. Lab. Carlsberg 11. cited from Winge (1929).

- (1929). On the nature of the sex chromosomes in Hurnulus. Hereditas 12. 